\title{
The effect of premolar occlusal contact on the occlusal forces
}

\author{
Moe Win Thein, Yoichiro Seki, Yukihiro Fujita \\ Orthodontic Science, Department of Orofacial Development and Function, \\ Division of Oral Health Sciences, Graduate School,
}

Tokyo Medical and Dental University

[Recieved: January 11, 2002.]

Key Word: Dental Prescale, Bite force, Occlusal contact, Premolars

\begin{abstract}
Clarifying the effects of changes in the number of occlusal contact points on occlusal function is important. In the present study, we measured occlusal forces using dental prescale films to ascertain the effects of changes in premolar occlusal contact. Subjects were seven adult males with normal occlusion. Subjects were instructed to bite a dental prescale film as hard as possible while using a maxillary bite plate (every mandibular tooth made contact with the plate), to measure occlusal forces. Next, the surface of each bite plate that came into contact with the mandibular premolars was ground so that mandibular premolars did not come into contact with the plate. Subjects were then asked to use the ground bite plate and repeat the test. The results were as follows: (1) when premolar occlusal contact was absent, the total occlusal force was significantly lower than that when premolar occlusal contact was present; (2) when premolar occlusal contact was absent, the level of occlusal forces applied to the molar region was significantly greater than that when premolar occlusal contact was present; and (3) in particular, when premolar occlusal contact was absent, the level of occlusal forces applied to the first molar region was significantly greater than that when premolar occlusal contact was present. Conversely, occlusal forces applied to the second molar demonstrated minimal change, irrespective of premolar occlusal contact. These findings suggest that premolar occlusal contact affects the distribution of occlusal forces applied to the other teeth. In particular, changes in premolar occlusal contact affect the level of occlusal forces applied to the neighboring first molar, and are involved in the expression of maximum occlusal forces.
\end{abstract}




\section{Introduction}

Numerous reports on occlusal forces and the neuromuscular mechanisms involved in generating these forces have been published ${ }^{1}$. Some reports have found that molar occlusion with left-to-right symmetry is necessary for the expression of maximum occlusal forces and masseter muscle activities ${ }^{2)}$, while other reports have documented a correlation between masseter muscle activity and the occlusal load center ${ }^{2-4)}$. According to previous research, the majority of occlusal forces are applied to the molar region ${ }^{4)}$. Furthermore, several studies have found that information from mechanoreceptors in the periodontal ligament regulates masseter muscle activity and is an important determinant of occlusal force ${ }^{5.6}$.
Nonetheless, data on the distribution of occlusal forces along the entire dentition has been difficult to record using conventional investigational methods. Although some studies have reported on occlusal forces applied to the molar regions, which bear the majority of applied occlusal forces, the contribution of each tooth to the expression of occlusal forces is not fully understood. Premolars are physically located close to the occlusal load center, but their roles have not been fully elucidated. The present study was conducted to clarify the occlusal function of each tooth by altering premolar occlusion using pressure-sensitive foils (Dental Prescale 50H), with which the occlusal function of each tooth could be assessed. a

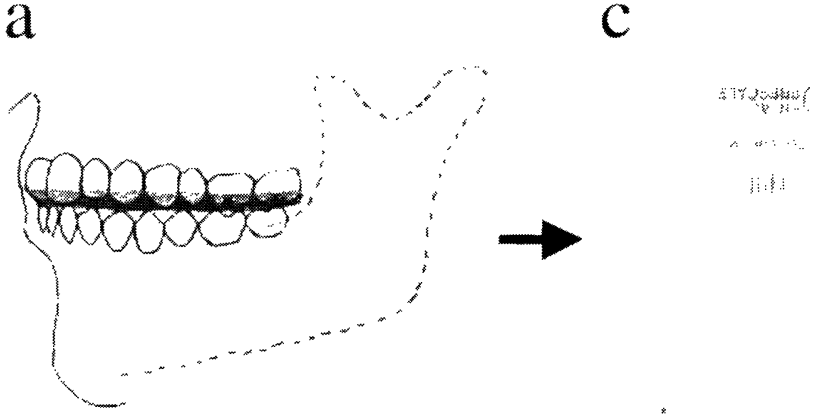

b

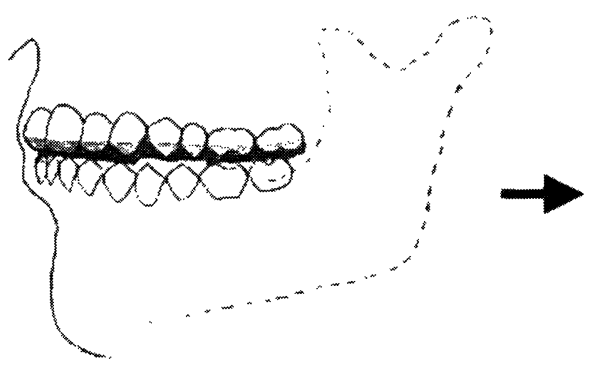

d

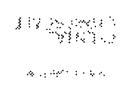

U
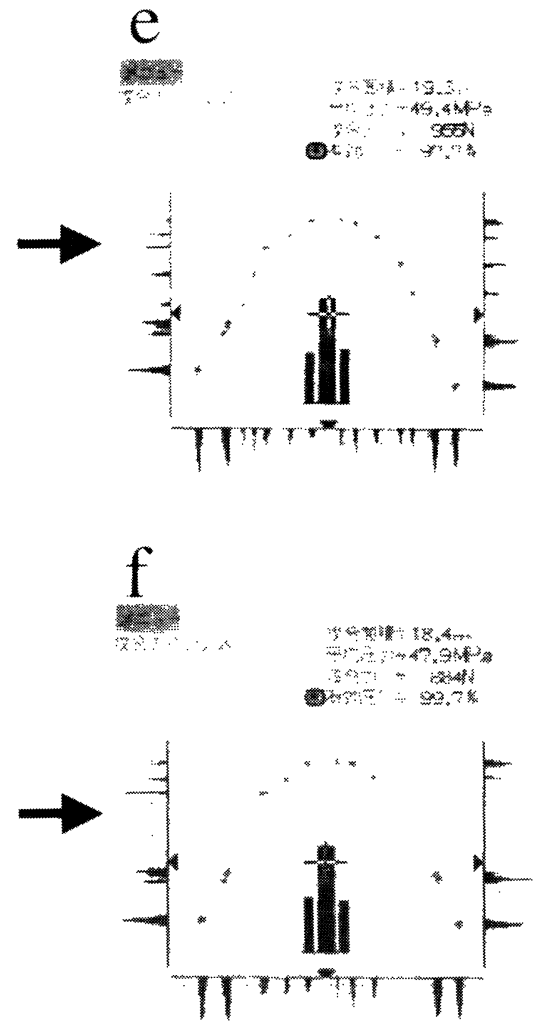

Fig. 1 a. The bite plate I was constructed to obtain the occlusal contacts in all regions. b. The bite plate II was remoulded by removing premolar occlusal contacts from bite plate I to exclude the premolar occlusal contacts. c. The Prescale foil showed occlusal contacts in all regions after biting as hard as possible with bite plate I. d. The Prescale foil showed the lack of occlusal contacts in premolar region after biting as hard as possible with bite plate II. e. The plastic film, indicated the occlusal contacts in all regions was printed out after scanned the Prescale foil taken with bite plate I by Occluzer. f. The plastic film, indicated the lack of occlusal contacts in premolar region was printed out after scanned the Prescale foil taken with bite plate II by Occluzer. 


\section{Materials and Methods}

\section{Subjects}

The subject group comprised seven males with normal occlusion and no present or past history of abnormal stomatognathic function. Subject age ranged from 25 years 8 months to 31 years 7 months, with a mean age of 27 years 8 months. The present study was performed only after obtaining informed consent from each subject.

\section{Preparation of bite plates}

In order to clarify the effects of premolar occlusion on occlusal functions in the present study, each subject wore a maxillary plate, with which occlusion could be easily altered by grinding the premolar region.

Using jaw trays and alginate impression materials, two impressions of the maxilla and one impression of the mandible were taken. Dental plaster was immediately poured into these impressions to prepare a working model. In addition, in order to attach the working model to an articulator, a bite pattern was taken using paraffin wax while adjusting the interocclusal distance in the first molar region to $2 \mathrm{~mm}$. Using a pressure molding machine (Biostar® SCHEU-DENTAL, Germany), a bite plate was prepared by softening and pressure molding a $1 \mathrm{~mm}$-thick plastic plate (IMPRELON "S", Ch.-No:1001A, SCHEU-DENTAL, Germany) to the maxillary working cast. The molded plate was then carefully removed from the working model while avoiding physical deformation, and was trimmed at the height of the center of the crown. Once a bite plate was properly constructed using the above wax bite pattern, the remaining working model was mounted to the articulator. The above-mentioned plate was placed on the maxillary model attached to the articulator and adjusted, so that the buccal cusp of every tooth (functional mandibular cusps) made contact with the plate using a quickcuring acrylic resin. The plate was then inserted in the oral cavity, and its position was adjusted using pressure-sensitive foils so that the occlusal force of each region (anterior tooth region, canine region, premolar region and molar region) closely resembled the data obtained from individuals with normal occlusion ${ }^{7)}$. In the present study, the term "Plate I" was used to describe a plate with which every functional mandibular cusp made contact with the plate (Figure 1a). Furthermore, after measuring maximum occlusal force using Plate I, the contact points between Plate I and the mandibular premolar cusps were ground using a stainless steel bar to pre pare Plate II, which describes a plate with no premolar occlusal contact (Figure 1b).

\section{Measurement of occlusal force}

While wearing Plate l, each subject was asked to sit deeply in a dental chair and stretch the back so that the FH plane was parallel to the floor. Each subject was then asked to bite several times to ensure plate stability. Occlusal functions were assessed using pressure-sensitive foils (Dental Prescale $50 \mathrm{H}$ type R, Fuji Film Co, Japan) (Figures $1 \mathrm{c}$ and 1d). The thickness of these films was $0.097 \mathrm{~mm}$, with a range of accuracy of 5-120 MPa. While wearing Plate I, a pressure-sensitive foils was inserted into the oral cavity, and each subject was instructed to bite the film as hard as possible for two seconds. This test was repeated every 30 minutes for a total of eight times. After measuring occlusal forces using Plate I, Plate II was prepared. Occlusal forces were then measured in the same manner for a total of eight times.

\section{Analysis}

Each film was placed back into the light protecting package immediately after testing, and was examined by a special analyzer (Occluzer FDP 703, Fuji Film Co, Japan) approximately one hour after testing (Figures le and 1f). The following three parameters were measured: total occlusal force $(\mathrm{N})$, occlusal force in different regions $(\mathrm{N})$, and occlusal load center $(\%)$. According to the method of Shinogaya et al. ${ }^{8)}$, occlusal load center was determined by analyzing the anteroposterior and left-right balance of the dental arch. A t-test and Wilcoxon signed rank test was used for statistically comparisons of the data obtained using Plates I and II, and values of $\mathrm{p}<0.05$ were considered statistical significant. In addition, the coefficient of variation was calculated for total occlusal force measurements made with Plates I and II, to assess the degree of measurement errors.

\section{Results}

\section{Total occlusal force}

Maximum occlusal force was measured eight times each for Plates I and II, for each of the seven subjects. As a result, a total of 112 tests were performed using pressure-sensitive foils. Figure 2 shows the total occlusal forces measured with Plates I and II. In all subjects, total occlusal force measured with Plate II was significantly smaller than that measured with Plate I. In addition, Table 1 shows the coefficient of variation for measurements made with Plates I and II in each subject. In all subjects, the coefficient of variation was low, ranging from $2.7-10.8 \%$. 


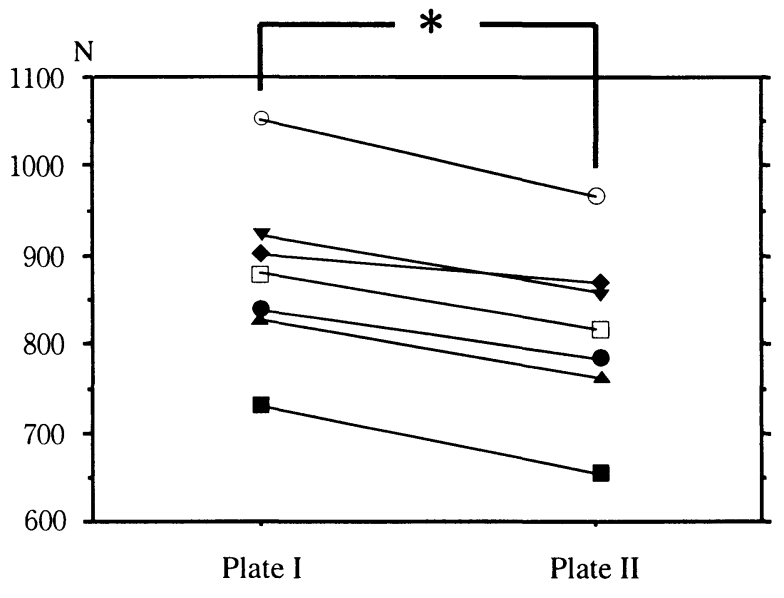

Fig. 2 Total occlusal forces distribution in Plate I and Plate II of each subject which indicated statistically significance $(\mathrm{P}<0.05)$.

Table $1 \mathrm{CV}$ values of total occlusal forces analysis (\%)

\begin{tabular}{ccc}
\hline Subject & Plate I $(\mathrm{n}=8)$ & Plate II $(\mathrm{n}=8)$ \\
\hline 1 & 7.6 & 9.0 \\
2 & 9.4 & 3.1 \\
3 & 10.8 & 4.4 \\
4 & 7.8 & 5.4 \\
5 & 4.1 & 4.6 \\
6 & 8.7 & 2.7 \\
7 & 6.7 & 3.3 \\
\hline
\end{tabular}

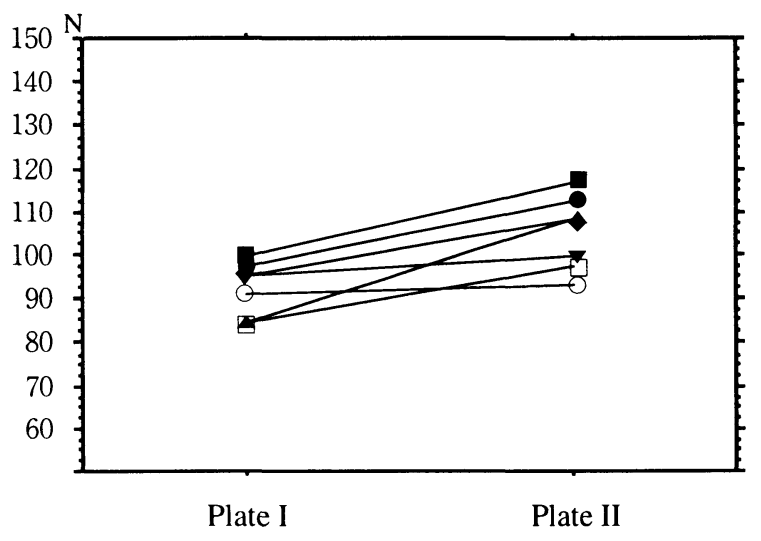

Fig. 3 Anterior regional occlusal forces distribution in Plate I and Plate II of each subject which indicated statistically no significance.

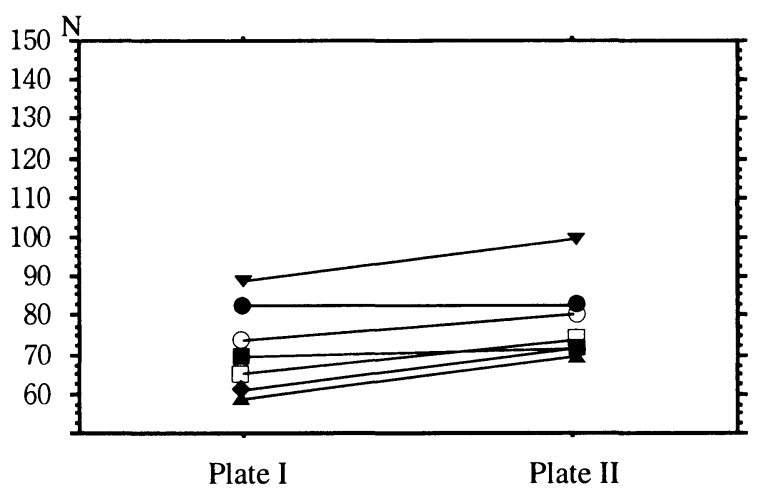

Fig. 4 Canine regional occlusal forces distribution in Plate I and Plate II of each subject which indicated statistically no significance.

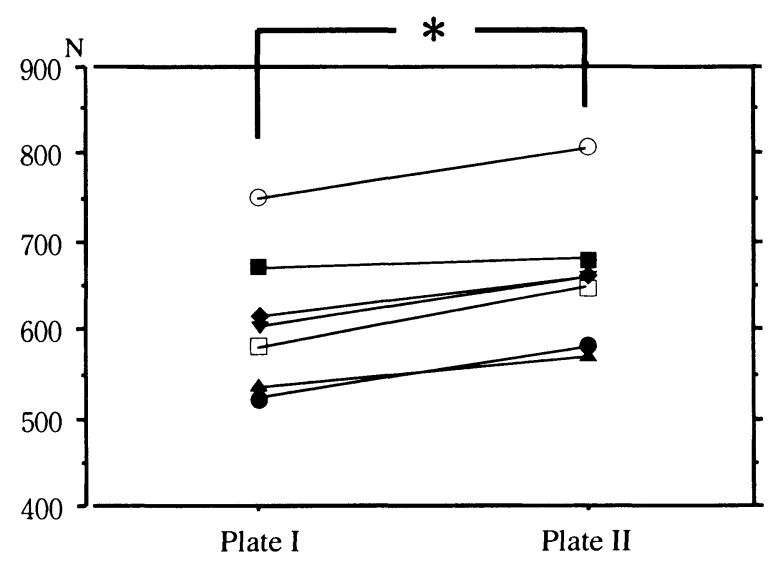

Fig. 5 Molar regional occlusal forces distribution in Plate I and Plate II of each subject which indicated statistically significance $(\mathrm{P}<0.05)$

\section{Occlusal forces in different regions}

Occlusal forces were measured in the various regions for each subject while wearing Plate I or II. Figure 3 shows mean occlusal force in the anterior tooth region (four teeth, from the left second incisor to the right second incisor); Figure 4, that in the canine region; Figure 5 , that in the molar region. The results demonstrated no significant differences in occlusal force in the anterior tooth or canine regions between Plates I and II. However, occlusal force in the molar region of Plate II was significantly higher than that in Plate I. As the majority of occlusal force is applied to the molar region, distribu- 


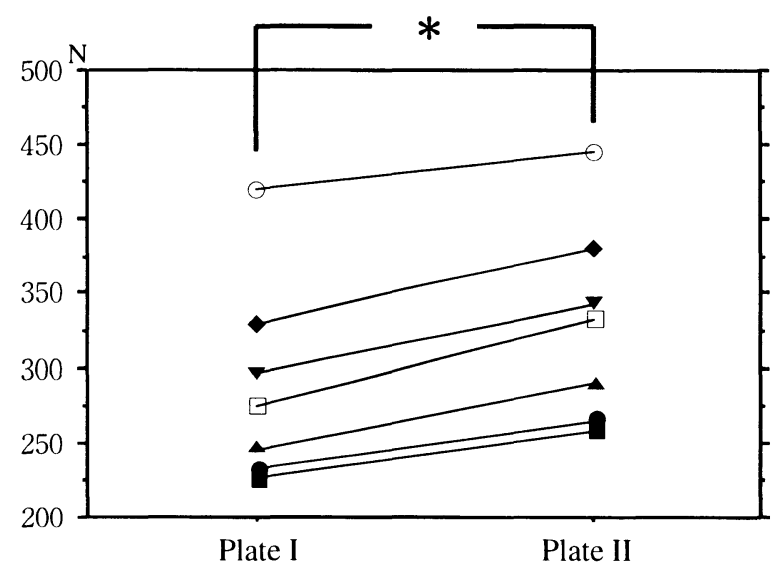

Fig. 6 First molar region occlusal forces distribution in Plate I and Plate II of each subject which indicated statistically significance $(\mathrm{P}<0.05)$.

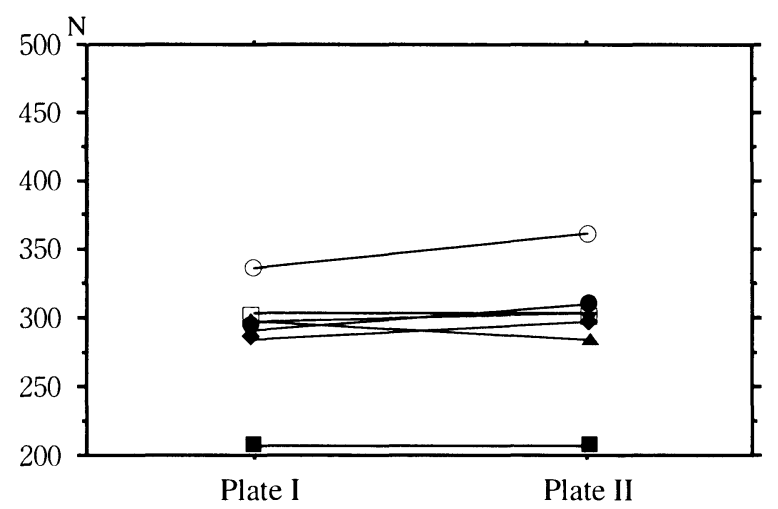

Fig. 7 Second molar region occlusal forces distribution in Plate I and Plate II of each subject which indicated statistically no significance.

tion of occlusal forces applied to the molar region was further analyzed at the first and second molars. Figure 6 shows mean occlusal force at the first molar; and Figure 7, that at the second molar. The results revealed that occlusal force at the first molar was significantly higher in Plate II than Plate I, but no significant differences in occlusal force were observed at the second molar between Plates I and II.

\section{Occlusal load center}

Table 2 shows the antero-posterior and left-right centers determined from Plates I and II, and the respective coefficients of variation. With Plate I, the left-right occlusal load
Table 2 Relative antero-posterior and right-left location of occlusal load center.

\begin{tabular}{lccccc}
\hline \hline & \multicolumn{2}{c}{ Plate I } & & \multicolumn{2}{c}{ Plate II } \\
\cline { 2 - 3 } \cline { 5 - 6 } & Width & Length & & Width & Length \\
\hline mean & $50.54 \%$ & $61.77 \%$ & & $51.16 \%$ & $62.37 \%$ \\
sd & 5.404 & 6.204 & & 5.630 & 5.644 \\
cv & $10.7 \%$ & $10.0 \%$ & & $11.0 \%$ & $9.0 \%$ \\
\hline
\end{tabular}

center was $50.54 \%$ of the total width, while the antero-posterior center was $61.77 \%$ of the total length. The coefficient of variation for antero-posterior and left-right occlusion centers was about $10 \%$, confirming that the antero-posterior and leftright occlusion centers remained stable. Furthermore, the coefficient of variation for the occlusal load center assessed with Plate II was low, confirming the stability of the plate.

\section{Discussion}

In the past, occlusal forces were assessed by measuring electromyographic activity of the masseter muscle "), measuring occlusal contact area ${ }^{1.2}$, or calculating mastication efficiency ${ }^{10.11)}$. Numerous electromyographical studies have been reported, and some have found that muscle activity is correlated with molar occlusion, but not with premolar occlusion ${ }^{1.4)}$. Nonetheless, few studies have closely investigated the relationship between occlusal contacts and function, and the effects of each tooth on occlusal function have not been fully elucidated. In order to measure occlusal forces in different regions within the dental arch, the present study therefore utilized pressure-sensitive foils (Dental Prescale 50H), which have been shown to assess close-to-natural occlusal forces with a high degree of reproducibility ${ }^{12)}$. Coefficients of variation were calculated for the various measurements made in the present study, and the results demonstrated that coefficients of variation were indeed low. Furthermore, in order to alter the premolar occlusal contact, each subject was asked to use a maxillary bite plate, to enable simple modification of occlusal contacts. Some of the problems associated with the use of these plates are unstable occlusal height and balance within the dental arch. In order to resolve the former problem, occlusal height in the molar region was set at $2 \mathrm{~mm}$, within the resting range for the mandible, to minimize fluctuations in the occlusal height. To address the problem of occlusal balance within the arch, Plate I was carefully adjusted in terms of total occlusal force and antero-posterior and 
left-right balance within the dental arch, based on data obtained from individuals with normal occlusion ${ }^{7)}$. According to a previous report using Dental Prescale $50 \mathrm{H}$, occlusal force in the molar region is about six times greater than that in the premolar region in individuals with normal occlusion ${ }^{8}$. However, in the present study, the large occlusal force at the molar region was attributable to early contacts at the molar region caused by the thickness of these pressuresensitive foils. As a result, each plate in this study was made by measuring occlusal forces at each tooth using pressure-sensitive foils to avoid early contact at the molar region. With regard to the balance of occlusal forces at each tooth, based on the results of a previous study ${ }^{7)}$, the occlusal force applied to the molar region was adjusted to about four times greater than that applied to the premolar region. By carefully performing these procedures, reproducibility of the antero-posterior and left-right balance within the dental arch improved. Furthermore, like past studies ${ }^{8)}$, the occlusal load center was established at the middle of the left-right dentition near the first molar. The results of occlusal load center analyses revealed that the coefficient of variation for the measurements made with Plate I was $10.0 \%$ for antero-posterior balance, and $10.7 \%$ for left-right balance, demonstrating that Plate I was stable during the eight maximum occlusal force measurements in each subject. In addition, the coefficient of variation for the measurements made with Plate II (Plate I with ground premolar region) was $9.0 \%$ for antero-posterior balance, and $11.0 \%$ for left-right balance, revealing that the tests were performed in a stable manner. By taking measurements using these plates, results were able to be obtained with a sufficient degree of reproducibility.

The present study investigated the effects of changes in occlusion, namely the presence and absence of premolar occlusal contact, on occlusal functions. The results demonstrated that total occlusal force without premolar occlusal contact was lower than that with premolar occlusal contact. The reason for this could be a reduction in the number of teeth supporting occlusal forces or a lack of input from mechanoreceptors on the periodontium of each tooth. The former agrees with the results of previous studies documenting the relationship between the number of teeth involved in occlusion and occlusal force ${ }^{11}$, whereas the latter could be explained in terms of suppressed muscle activity due to a loss of mechanical stimuli from the premolar region. Several studies have confirmed that occlusal forces are regulated by comprehen- sive processing of data sent from mechanoreceptors present in the periodontium ${ }^{13-15)}$. When premolar occlusion was eliminated, data that are normally transmitted from the premolar region to the central nervous system were eliminated as well, changing the physiological functions involved in occlusal function.

Figures 2 through 7 show findings associated with occlusal forces in each region. No significant differences in occlusal force were observed in the anterior tooth region between Plates I and II. Occlusal force in the canine region displayed increased values without premolar occlusal contact, but this increase was not significant. Morphologically and functional$l y$, the anterior tooth and canine regions are considered areas that do not bear occlusal forces. In fact, the ratio of occlusal forces applied to these regions is very small in individuals with normal occlusion. It is reasonable to assume that the effects of changes in premolar occlusal contact become less significant further away from the occlusal load center. Furthermore, occlusal force in the molar region of Plate II was greater in all subjects than that of Plate I. The molar region is considered an important area for bearing occlusal forces ${ }^{4,16.17)}$, and this region closely correlates to the muscle activity of the masseter muscle. Morphologically, the capacity of the molar region to bear loads in the direction of occlusal force should be great. As a result, the amount of occlusal force applied to the molar region was analyzed at both the first and second molars. The results demonstrated that occlusal force at the first molar in Plate II was significantly higher than that in Plate I, whereas no marked changes were observed in occlusal force at the second molar between Plates I and II. One previous study has reported that individual biting force (i.e., occlusal force load bearing capacity in the periodontal tissue) was greatest for the first molar ${ }^{18)}$. In addition, when compared to the second molar, the first molar is physically closer to the occlusal load center. In the present study, few differences existed in the occlusal load center between Plates I and II (or in other words, before and after grinding the premolar occlusion contact points). This increase in occlusal force at the first molar is thus a result of the first molar bearing a greater load of occlusal forces despite the absence of premolar occlusal contact.

When all occlusion contact points are intact, the maximum occlusal force is generated by each tooth expressing maximum occlusal force. Since the loss of premolar occlusal contact resulted in reduced total occlusal force and increased 
molar occlusal force, at the first molar in particular, the occlusal load at the first molar might become functionally excessive without support from the premolar region. Conversely, when occlusal loads are distributed throughout the dentition, each tooth expresses an appropriate amount of occlusal force, and contraction of the masseter muscle is controlled by comprehensive analysis of the data sent from each tooth ${ }^{1.4)}$. However, when input from the premolar region is missing, the data input system is disrupted, increasing the amount of load placed on the first molar region and reducing total occlusal force. Although the upper limit for bearing occlusal force for each tooth has not been clarified, the results of the present study suggest that the distribution of occlusal force within the dental arch, and premolar occlusal contact in particular, is closely correlated with the expression of maximum occlusal force.

\section{Acknowledgements}

The authors are grateful to Prof. Kunimichi Soma (Orthodontic Science, Tokyo Medical and Dental University) for his continued kind instruction. The authors thank the staffs in the department for their comprehensive support and valuable comments. This work was partly supported by Grants-in-Aids for Scientific Research (No.12307050, 11470448) from the Ministry of Education, Culture, Sports, Science and Technology of Japan.

\section{References}

1 ) Bakke, M.: Mandibular elevator muscles: physiology, action, and effect of dental occlusion, Scand J Dent Res, 101: 314-331, 1993.

2 ) Hidaka, O., Iwasaki, M., Saito, M., et al.: Influence of clenching intensity on bite force balance, occlusal contact area and average bite pressure, J Dent Res, 7: 1336-1344, 1999.

3 ) Watkinson, A. C.: Electromyographic biofeedback and sustained masseter muscle contraction in man, $\mathrm{J}$ Oral Rehabil, 15: 353-359, 1988.

4 ) Manns, A., Miralles, R., Valdivia, J. et al.: Influence of variation in anteroposterior occlusal contacts on electromyographic activity, J Prosthet Dent, 61: 617-23, 1989.

5 ) Steenberghe, D. V.: The structure and function of periodontal innervation, J Periodontal Res, 14: 185-203,
1979.

6 ) Berkovitz, B. K. B., Moxham, B. J and Newman, H. N.: The periodontal ligment in health and disease, 133-181, Second Edition.

7 ) Jenkins, G. N.: Mastication and deglutition,edited by Jenkins, G, N., The physiology and biochemistry of the mouth, 518-521, Oxford. Blackwell Scientific Publications, 1978.

8 ) Shinogaya, T., Sodeyama, A. and Matsumoto, M.: Bite force and occlusal load distribution in normal complete dentitions of young adults, Eur J Prosthodont Rest Dent, 7: 65-70, 1999.

9 ) Haraldson, T., Carlsson, G. E., Dahlstrom, L. et el.; Relationship between myoelectric activity in masticatory muscles and bite force, Scand Dent Res, 93: 539-545, 1985.

10) Mioche, L and Peyron, M. A.: Bite force displayed during assessment of hardness in various texture contexts, Archs Oral Biol, 40, 415-423, 1995.

11) Hatch, J. P., Shinkai, R. S. A., Sakai, S., et al.: Determinants of masticatory performance in dentate adults, Arches Oral Biol, 46: 641-648, 2000.

12) Sodeyama, A., Shinogaya, T and Matsumoto, M.:Reproducibility of maximal bite force distribution over dentition, J Stomatol Soc Jpn, 65: 339-343. 1998

13) Klineberg, I and Murray, G.: Osseoperception: Sensory function and proprioception, Adv Dent Res, 13:120-129, 1999.

14) Bakke, M., Michler, L and Moller, E.: Occlusal control of mandibular elevator muscles, Scand. J Dent Res, 100:284-291, 1992.

15) Anderson, D. J., Hannam, A. G and Matthews, B.: Sensory mechanism in mammalian teeth and their supporting structures, Physiol Rev, 50: 171-195, 1970.

16) Kerstein, R. B.: Understanding and using the center of force, Dentistry Today, 116-119, 1998.

17) Baba, K., Tsukiyama, Y., Clark, G. T.: Reliability, validity, and utility of various occlusal measurement methods and techniques, J Prosthetic Dent, 83: 83-89, 2000.

18) Takamizawa, T. : Studies on the Co-relative and Individual Bite Forces of Normal Permanent Teeth, J Jpn Prosthodont Soc, 9: 217-236, 1965 